Cahiers $d u$ MONDE RUSSE

\section{Cahiers du monde russe}

Russie - Empire russe - Union soviétique et États indépendants

$53 / 4 \mid 2012$

Varia

\title{
Evgeny Dobrenko, Galin Tixanov, eds., A History of Russian Literary Theory and Criticism
}

\section{Catherine Depretto}

\section{OpenEdition}

\section{Journals}

Édition électronique

URL : http://journals.openedition.org/monderusse/7805

DOI : $10.4000 /$ monderusse. 7805

ISSN : $1777-5388$

\section{Éditeur}

Éditions de l'EHESS

\section{Édition imprimée}

Date de publication : 15 décembre 2012

ISSN : $1252-6576$

\section{Référence électronique}

Catherine Depretto, « Evgeny Dobrenko, Galin Tixanov, eds., A History of Russian Literary Theory and Criticism », Cahiers du monde russe [En ligne], 53/4 | 2012, mis en ligne le 07 octobre 2013, Consulté le 25 septembre 2020. URL : http://journals.openedition.org/monderusse/7805 ; DOI : https://doi.org/ 10.4000/monderusse.7805

Ce document a été généré automatiquement le 25 septembre 2020.

(c) École des hautes études en sciences sociales 


\title{
Evgeny Dobrenko, Galin Tixanov, eds., A History of Russian Literary Theory and Criticism
}

\author{
Catherine Depretto
}

\section{RÉFÉRENCE}

Evgeny DoBRenKo, Galin TIXANov, eds., A History of Russian Literary Theory and

Criticism. The Soviet Age and Beyond. Pittsburgh : The University of Pittsburgh

Press, 2011, 406 p.

1 Depuis le milieu du XIX ${ }^{\mathrm{e}}$ siècle, la critique littéraire tend à occuper une place importante dans la vie intellectuelle russe. Qu'il s'agisse de la critique journalistique (publicistika) comme de l'histoire de la littérature (literaturovedenie), elle a contribué à la constitution d'une sphère publique, jouant un rôle proche de celui d'une tribune de discussion et constituant l'un des éléments du fameux littéraro-centrisme russe. À la période soviétique, dégel et perestroïka exceptés, la critique a, en revanche, surtout été l'une des composantes du dispositif politique de contrôle de la littérature et de la culture.

2 Jusqu'à présent on ne disposait pas d'études d'ensemble de la critique littéraire russe au Xxe siècle, même si, depuis 2000 en particulier, étaient parus des ouvrages consacrés à certains aspects du sujet, quelques recueils de documents ou des manuels universitaires. Aussi l'ouvrage collectif ${ }^{1}$ proposé par deux spécialistes renommés de la culture russe, Evgeny Dobrenko, professeur à l'université de Sheffield et Galin Tixanov, titulaire de la chaire George Steiner de littérature comparée à Queen Mary, University of London, aidés d'une équipe internationale brillante ${ }^{2}$ vient-il d'abord combler une lacune importante.

Comme pour le Cambridge Companion consacré à la littérature russe $d u x^{e}$ siècle, dû à Evgeny Dobrenko et Marina Balina ${ }^{3}$, le livre sur la critique se veut novateur ; il intègre, par exemple, l'activité de l'émigration et englobe la période qui suit l'effondrement de 
l'U.R.S.S. Il ne laisse de côté aucune des facettes d'une activité considérée aussi bien à travers ses textes que dans sa dimension institutionnelle.

4 Cependant, ce programme ambitieux débouche sur un découpage en chapitres qui suit finalement d'assez près les divisions classiques de l'histoire soviétique et postsoviétique: la révolution et la guerre civile, la NEP, la révolution culturelle 1928-19324, les années trente, la guerre, l'après-guerre, le dégel, les années 1970, la perestroïka et au-delà, sans essayer de proposer une périodisation qui pourrait être propre à une histoire de la critique comme de la théorie littéraire ${ }^{5}$. Cette périodisation "classique" tient sans doute au caractère pionnier de l'entreprise et aussi à l'imbrication - encore plus grande que pour la littérature - de la critique et de la politique, ce qui, selon les périodes, lui donne un rôle plus prescriptif ou plus contestataire. Mais, dans tous les cas, son lien avec le pouvoir semble plus fort que pour la création, d'où cette référence systématique au contexte politique dont il est impossible de faire l'économie.

5 L'ampleur du champ que prétend couvrir l'ouvrage pour une période aussi longue impose, en outre, une tension permanente entre une approche strictement chronologique, traitant de tous les aspects du sujet pour une période donnée et une approche thématique, isolant, pour chaque période, tel ou tel secteur. Le fait que chaque sujet ait été confié à un ou deux contributeurs exclut un traitement continu des différents aspects de la question pour l'ensemble de la période concernée et, malgré un travail d'uniformisation, les différences d'approche et de perspective ne sont pas totalement gommées, sans compter que les découpages chronologiques ne sont pas les mêmes pour les différents sujets ${ }^{6}$. Compte tenu de l'absence d'une conceptualisation d'ensemble de l'histoire culturelle de la Russie soviétique, il n'était guère possible de procéder autrement. Le parti pris consistant à donner des chapitres cohérents sur des sujets particuliers était sans doute le plus réaliste. Si l'on perd en termes de continuité narrative, on y gagne en diversité et originalité des approches. De fait, l'ouvrage, même s'il embrasse une masse impressionnante de matériaux et couvre pratiquement tous les sujets relevant de l'histoire de la critique littéraire en terrain russe au $\mathrm{xx}^{\mathrm{e}}$ siècle, ne peut prétendre à l'exhaustivité. Par sa conception il est sans doute plus destiné à être utilisé comme une encyclopédie que comme une monographie; il réunit des articles que l'on peut consulter de façon indépendante (usage largement facilité par l'index) et qui serviront de base précieuse aux historiens de la culture et de la littérature russes du $\mathrm{xx}^{\mathrm{e}}$ siècle.

6 Pour finir, nous voudrions mettre l'accent sur les aspects de l'ouvrage qui nous semblent les plus intéressants et formuler quelques remarques générales. D'abord le livre restitue la plupart des grandes discussions politico-critiques des années 1920-1930 (chapitres 2 et 3) qui, pour nombre d'entre elles, ont commencé à sombrer dans l'oubli. Il expose de façon convaincante le phénomène du "réalisme socialiste » $:$ il analyse les mécanismes de ce dispositif esthético-politique, en rappelant les principales étapes qui en ont fixé le canon jusqu'à la mort de Stalin (chapitres 5 et 8), puis en montrant la lente dégradation du dogme jusqu'à la fin de l'U.R.S.S. Il offre surtout un panorama presque complet de l'histoire des courants de théorie de la littérature (chapitres 4, 6, 11). Il s'intéresse à l'œuvre des grands noms de la science russe du $\mathrm{xx}^{\mathrm{e}}$ siècle, mais n'oublie pas des entreprises moins célèbres, comme les travaux de l'antiquisante, cousine de Boris Pasternak, Olga Frejdenberg (1890-1955) ou ceux de l'élève des formalistes, Lidija Ginzburg (1901-1990). Il n'occulte pas les courants marxistes et 
sociologiques, pas plus que la sémantique de Nikolaj Marr (1864-1934). Signalons, en particulier, l'inventivité de Caryl Emerson qui a choisi d'illustrer les différences entre ce qu'elle identifie comme les quatre courants principaux de théorie de la littérature dans les années 1920 (formalistes, cercle de Bahtin, critique marxiste, psychanalytique) à partir de l'étude d'un cas précis, l'interprétation de l'œuvre de Gogol'. Tout aussi convaincant est le chapitre de Katerina Clark et Galin Tixanov, consacré aux théories esthétiques des années trente et aux débats autour du genre romanesque (Bahtin, Luká cs). L'ouvrage mène aussi l'étude jusqu'à la période la plus actuelle et offre des pistes intéressantes pour comprendre l'évolution récente de la critique en Russie (chapitres 14-15).

7 Même si la recherche savante est bien représentée par l'étude de ses figures majeures, on pourra toutefois regretter l'absence d'un chapitre consacré à la critique universitaire en tant que telle dont l'importance est centrale pour toute la période et qui a su, en dépit de certaines adaptations (cf. le terme d' "akademičeskij marksizm " proposé par A. Dmitriev), préserver un socle de compétences et réaliser de véritables avancées, dans le domaine de l'édition des classiques en particulier. Dans un autre ordre d'idée, l'aspect proprement institutionnel de la critique, affirmé pourtant comme une de ses caractéristiques à la période soviétique ${ }^{7}$, n'est pas assez systématiquement mis en évidence ${ }^{8}$ : on trouve en définitive assez peu de chose sur l'insertion de la critique dans le dispositif de l'Union des écrivains ou sur la participation des critiques aux processus de mise en conformité idéologique comme sur leur rôle dans les périodes de «libéralisation». Parfois, l'objet de l'ouvrage, la critique, se confond tout simplement avec l'histoire de la littérature en général et son rôle spécifique tend à se diluer dans l'évocation générale de la culture.

Il n'en reste pas moins qu'il faut saluer comme il le mérite ce livre magistral, point d'aboutissement de longues années de recherche et fruit d'un travail attentif de coordination, qui constitue d'ores et déjà un ouvrage de référence.

\section{NOTES}

1. Une édition parallèle en russe est parue la même année, avec toutefois des différences, non dans la conception d'ensemble du volume, mais dans la teneur de certains articles, beaucoup plus conséquents en volume, cf. ЕВГЕНИЙ ДОБРЕНКО, ГАЛИН ТИХАНОВ РЕД., ИСТОРИЯ РУССКОЙ ЛИТЕРАТУРНОЙ КРИТИКИ: СОВЕТСКАЯ И ПОСТСОВЕТСКАЯ ЭПОХИ, М.: « Novoe literaturnoe obozrenie ", 2011, 792 p. Les chapitres 11 et 12 ne se trouvent pas dans le même ordre.

2. Par ordre de participation, Stefano Garzonio, Maria Zalambani, Natalia Kornienko, Caryl Emerson, Hans Günther, Katerina Clark, Ilya Kalinin, Mark Lipovetsky, Mikhail Berg, William Mills Todd III, Birgit Menzel, Boris Dubin, Catharine Theimer-Nepomnyashchy, Ilya Kukulin, Nancy Condee, Eugeniia Kupsan.

3. Evgeny Dobrenko, Marina Balina, eds., The Cambridge Companion to Twentieth-Century Russian Literature, Cambridge : Cambridge University Press, 2011.

4. Variante pour d'autres sujets : années vingt, années trente ou encore entre-deux-guerres. 
5. Il n'est pas certain, par exemple, qu'il soit indispensable de séparer les années 1917-1921 de la période 1921-1927, en confiant les deux chapitres à des auteurs différents.

6.

7. Cf. l'introduction signée E. Dobrenko et G. Tixanov, « Toward a History of Soviet and PostSoviet Literary Theory and Criticism ».

8. Cela tient incontestablement à l'absence d'une véritable histoire sociale de l'intelligentsia à la période soviétique, comme au parti pris des responsables du volume de ne pas trop tenir compte des publications de documents d'archives, concernant les rapports entre le pouvoir et la culture ou plus généralement des travaux d'historiens sur les phénomènes d'opinion et d'évolution des mentalités (Elena Zubkova pour l'après-guerre par exemple). 